Crime, Histoire \& Sociétés / Crime, History \& Societies

Vol. 21, n² | 2017

L'histoire de la criminalité et de la justice pénale : propositions de recherche pour le $21^{\mathrm{e}}$ siècle

\title{
Spatial turn et histoire de la justice pénale moderne
}

Falk Bretschneider

\section{(2) OpenEdition \\ Journals}

Édition électronique

URL : http://journals.openedition.org/chs/1994

DOI : 10.4000/chs.1994

ISSN : 1663-4837

Éditeur

Librairie Droz

Édition imprimée

Date de publication : 31 décembre 2017

Pagination : 297-307

ISSN : $1422-0857$

\section{Référence électronique}

Falk Bretschneider, «Spatial turn et histoire de la justice pénale moderne », Crime, Histoire \& Sociétés / Crime, History \& Societies [En ligne], Vol. 21, n² | 2017, mis en ligne le 19 juillet 2020, consulté le 16 janvier 2021. URL : http://journals.openedition.org/chs/1994 ; DOI : https://doi.org/10.4000/chs.1994 


\title{
Spatial turn et histoire de la justice pénale moderne
}

\author{
Falk Bretschneider
}

$\mathrm{R}$ éfléchir aux rapports historiques entre espace et justice pénale ressemble à la fois à une belle banalité et un véritable défi. Banalité, puisque l'action judiciaire, comme toute pratique humaine, s'inscrit dans et se nourrit de la structuration spatiale du social. Pour s'en convaincre il suffit de se rappeler qu'elle se calque sur l'un des principes d'organisation de l'espace les plus structurants, à savoir l'opposition entre mondes urbains et mondes ruraux. Malgré un intérêt grandissant pour les «justices de village », notre image de la justice pénale reste profondément urbaine : c'est dans les villes que se joue le grand théâtre de la justice, qu'il s'agisse là de la conséquence d'une différence réelle ou du simple effet de la documentation historique qu'elles abritent, comparativement plus riche que celle du plat pays ${ }^{1}$. C'est ici que siègent la plupart des tribunaux compétents en la matière, à proximité des hauts lieux du pouvoir politique auxquels ils se mélangent parfois, et c'est également ici qu'ont lieu les mises en scènes les plus spectaculaires de la pénalité ancienne. Or, bien qu'étant évident, ce lien étroit entre structuration de l'espace et activité judiciaire reste toujours pour l'essentiel inscrit dans un angle mort de l'historiographie, ce qui ne facilite pas la tâche de celui qui tente de le circonscrire. Ainsi, toute recherche bibliographique combinant les termes «histoire», «justice» et «espace» débouche sur des résultats plutôt maigres (quantitativement parlant ${ }^{2}$ ) dont une grande partie concerne d'ailleurs un champ très dynamique des études actuelles en géographie: la réflexion autour de la spatialisation des inégalités sociales, ou la «justice spatiale» - par exemple l'accès différencié à des services publics comme l'institution judiciaire ${ }^{3}$. Dans le domaine qui nous intéresse plus particulièrement ici, l'histoire de la justice pénale, l'espace n'est en revanche pour l'instant que très rarement questionné de manière explicite, c'est-à-dire en ayant recours à la notion non seulement pour décrire et mobiliser une catégorie de perception fondamentale du quotidien des acteurs, mais plus largement pour en faire une catégorie d'analyse permettant de saisir ce que d'autres n'ont pas encore permis d'apercevoir.

Brizay et al. (2002); aussi Frank (1995); Chauvaud et al. (2001).

2 Un autre indice est l'absence total du terme «espace/space», en dehors d'un usage trivial, dans les titres des contributions de notre revue.

3 Numéro «Justice spatiale» des Annales de géographie (2009); Bret et al. (2010).

Crime, Histoire \& Sociétés / Crime, History \& Societies 2017, vol. 21, n 2, pp. 297-307. 


\section{UN NOUVEAU REGARD SUR L'ESPACE}

Ce désintérêt, ou plutôt cette indifférence, des historiens de la justice pénale envers l'espace peut de prime abord surprendre, tant il va à l'encontre de l'un des courants les plus puissants du renouveau de l'historiographie internationale dans les dernières années: le «tournant spatial» ou spatial turn ${ }^{4}$. Loin de se résumer à un objet clairement identifiable, une théorie ou une méthode, ce terme désigne d'abord, plus modestement, une attention nouvelle portée à l'espace comme objet et catégorie d'analyse au sein d'une discipline dont la catégorie directrice a si longtemps été le temps $\mathrm{s}^{5}$. Toutefois, ce regain d'intérêt ne concerne pas toutes les historiographies de manière égale. Frère jumeau de la théorie postcoloniale, il est né au milieu des années 1980 aux États-Unis, dans le contexte d'une mise en question radicale (d'abord chez les géographes puis chez les tenants des autres sciences sociales) des hiérarchies établies entre centres et périphéries et des rapports de pouvoir produits par elles, au niveau local comme à l'échelle globale ${ }^{6}$. En Europe, cette nouvelle approche a parfois été accueillie avec un enthousiasme démesuré, par exemple en Allemagne où elle a permis de se libérer de l'opprobre jeté sur la notion d'espace après son instrumentalisation idéologique par les nazis (et son usage tout à fait réel au service d'une politique destinée à conquérir un nouvel «espace vital» à l'est) ${ }^{7}$; mais parfois elle a aussi été superbement ignorée ou reçue avec condescendance, notamment par les historiens français qui ne ressentaient guère la nécessité de s'interroger davantage sur l'espace après tant de débats - favorisés par la communauté de formation avec les géographes - qui avaient vu s'affronter souvent les meilleurs de la discipline (comme LucienFebvre et FernandBraudel, entre «possibilismes» et «déterminismes» spatiaux, positions réconciliées plus tard par BernardLepetit dont les travaux ont débouché sur la proposition d'un nouvel axiome permettant de réunir, dans un même mouvement d'analyse, structures spatiales et modalités d'action humaine: celui des échelles) ${ }^{8}$.

Contrairement à ce que le terme «tournant» évoque, il ne s'agit donc pas d'un mouvement général ou d'un paradigme unanimement partagé; c'est plutôt une perspective, adoptée ou non par l'observateur, et pouvant se traduire, selon chaque cas concret, par un intérêt envers les représentations spatiales, la production de l'espace ou les expériences que les acteurs en font en lui conférant du sens. C'est peut-être dans cette diversité des conceptions et des applications qu'il convient de

4 Le constat est le même pour le domaine de l'histoire de la criminalité où une géographie historique du crime n'en est qu'à ses débuts, voir Denys (2016, p. 89).

5 Nous nous contentons ici de renvoyer aux remarques tout à fait lucides faites à ce propos par Morsel (2006).

6 Warf et Arias (2009); pour un résumé français de ces débats, voir Jacob (2014). Le site http://spatial scholarslab.org/spatial-turn/, proposé par l'historienne américaine JoGuldi, offre un aperçu enrichissant des enjeux du spatial turn dans plusieurs disciplines dont l'histoire.

7 Voici seulement quelques références majeures: Bachmann-Medick (2006); Döring et Thielmann (2008); Rau (2008). Pour un résumé français de la discussion autour du spatial turn dans l'historiographie allemande, de nature davantage théorique qu'empirique, et avec un accent mis sur les études médiévistes, voir Zotz (2006).

$8 \quad$ Il est inutile de redonner ici les références détaillées de ces débats; nous renvoyons aux dernières interventions de Lepetit (1999) et à la synthèse de Bourdelais et Lepetit (1986). Évidemment, il faut ajouter à ce panorama la tradition importante de l'histoire régionale qui marqua profondément l'historiographie française de l'après-guerre. Pour la discussion autour des échelles, l'ouvrage de Revel (1999) reste central. 
chercher l'une des raisons pour lesquelles les historiens du champ pénal - habitués à travailler avec des propositions plus cohérentes (la théorie foucaldienne, le processus de civilisation éliasien, etc.) - restent pour l'instant plutôt frileux face à l'espace. Car entre une conception traditionnelle et naïve qui continue à le considérer simplement comme le monde physique qui nous entoure, et les élaborations théoriques les plus audacieuses, souvent intéressées par des phénomènes spatiaux dématérialisés, virtuels et éphémères tels qu'ils se produisent à travers les contacts protéiformes s'établissant entre les membres d'une société de migration mondialisée', il est aisé de se perdre. Partons donc d'une définition relativement simple et qui conçoit l'espace d'abord comme un ensemble de relations concrètes se tissant entre les acteurs et les objets de leur environnement, en ne le réduisant ni à sa matérialité, ni à ses représentations, ni aux discours qui se forment autour de lui ${ }^{10}$. Il s' agit par conséquent de le penser comme une construction cognitive produite et perpétuée par un agir social en interaction avec les structures du monde physique (qu'il produit, mais qui crée et façonne, à son tour, des comportements humains) et de le saisir dans ses dynamiques en s'interrogeant sur les rapports qu'il entretient avec la justice pénale à l'époque moderne - rapports de productions, de pratiques ou d'appropriations spatiales ${ }^{11}$.

\section{PRODUIRE L’ESPACE : JUSTICE PÉNALE ET ESPACE TERRITORIAL}

La capacité de la justice pénale à créer l'espace apparaît peut-être le plus clairement au niveau de sa participation à la construction spatiale du politique. Puisque son exercice comptait partout dans l'Europe moderne au nombre des droits régaliens les plus nobles, elle était intrinsèquement liée à la définition du pouvoir politique et de la forme spatiale qui était la sienne, le territoire. Prenons un exemple: en 1539, le conseil de Lippstadt fit savoir au gouverneur de Clèves, capitale de l'un des trois duchés unis de Juliers-Clèves-Berg, que des émissaires du prince-évêque de Münster, voisin septentrional, avaient érigé plusieurs roues garnies de cadavres de criminels exécutés près d'une grande route, située sur la frontière entre les deux territoires et objet de leurs convoitises mutuelles. Les Lippois, alliés au duché, décidèrent de lancer ces roues dans la petite rivière qui coulait à côté, ce qui amena les Münsterois à en ériger immédiatement deux autres, elles aussi jetées à l'eau par leurs adversaires ${ }^{12}$. À première vue, cette compétition macabre dans un coin perdu du nord-ouest du Saint-Empire germanique paraît totalement absurde. Elle ne l'était pourtant pas. L'effort des deux côtés s'inscrivait en fait dans la rivalité que se livraient les membres de l'Empire autour de toutes sortes de droits, privilèges et prérogatives, servant de monnaie courante sur le marché de l'influence et de la gloire d'une société nobiliaire. Dans la querelle entre Münster et les ducs de Juliers-Clèves-Berg, la pomme de la discorde était la supériorité sur la route en question (et les droits de péage qui allaient avec), pour l'instant en possession des ducs, mais revendiquée par les Münsterois.

\footnotetext{
9 Soja (1996).

10 Löw (2015).

11 Bourdelais et Lepetit (1986, p. 23).

12 Schuster (2015, p. 252-253).
} 
Afin d'affirmer ces prétentions, placer près d'elle quelques roues de supplices symboles par excellence de la justice pénale et des pouvoirs régaliens afférents - avec au sommet, pour renforcer l'effet, les dépouilles de quelques pauvres condamnés, constituait par conséquent un geste puissant pour faire savoir aux autres que l'on souhaitait procéder à une redéfinition des rapports de force existants.

Cet exemple nous apprend donc que des roues de supplice pouvaient servir à d'autres finalités que la mise à mort des malfaiteurs. Il montre, de manière spectaculaire, ce qui était une évidence dans tout l'Empire: l'affirmation d'un pouvoir territorial y compris dans ses dimensions spatiales - passait aussi, et surtout, par l'exercice de la justice pénale ${ }^{13}$. Chaque exécution publique d'une peine se transformait ainsi en un acte performatif traduisant les prétentions politiques et sociales de celui au nom duquel il était accompli. C'est pourquoi il n'était pas rare, surtout dans les contrées les plus fragmentées de l'Empire, de voir une potence érigée à la lisière de deux juridictions ${ }^{14}$ - une pratique destinée à définir et délimiter l'espace juridique et politique, et qui commence également à susciter l'intérêt de la recherche en France, à travers l'étude interdisciplinaire des gibets et fourches patibulaires, à la fois instruments de justice mais aussi marqueurs des limites juridictionnelles et donc « du jeu plus politique de leurs détenteurs $»^{15}$. S'il «n'est pas de société qui ne développe son propre espace $»^{16}-$ et s'il convient par conséquent d'inscrire, afin d'étudier la contribution de la justice pénale à la spatialisation du politique, chaque cas dans son contexte particulier - on peut donc supposer l'existence de pratiques communes, ou du moins semblables, au sein de cette Europe moderne plurielle dont les différentes formes d'organisation politique (monarchie «absolutiste» en France, souveraineté partagée dans le Saint-Empire) sont si souvent pensées comme irréductibles les unes aux autres. Cela vaut évidemment aussi pour d'autres modalités de la production de l'espace, comme le tracé des routes et son influence sur l'emplacement des lieux de haute justice (à moins que ce ne soit l'inverse) ${ }^{17}$.

13 L'histoire de la justice pénale en Allemagne, dominée très largement par des études régionales, fait généralement écran à cette signification spatiale du territoire, en passant ainsi à côté de quelque chose d'essentiel, à savoir une organisation spatiale des liens sociaux qui prenait une forme fractale. Voir Bretschneider et Duhamelle (2016) et Bretschneider (2018).

14 Bellabarba (2002).

15 Charageat et Vivas (2015, citation paragraphe9); Bubenicek (2015) rapporte plusieurs cas qui se rapprochent de celui que nous avons cité, intervenus à la fin du XIV siècle et réunis sous le nom de «guerre des gibets» dans le comté de Bourgogne; pour l'Allemagne, voir Auler (2008-2012) dont ces travaux s'inspirent en partie.

16 Morsel (2006, p. 94).

17 De nombreux travaux soulignent la proximité entre gibets et voies de communication, en interprétant curieusement cette situation géographique souvent comme une preuve de leur mise à l'écart de la société. Mais aucune étude, à notre connaissance, ne s'est pour l'instant penchée de plus près sur le rapport entre lieux d'exécution et réseau routier en l'interrogeant en tant que tel. Certains - par exemple Spierenburg (1984, p.57-58) ou Dyndor (2015) - ont souligné le fait qu'une position près des grandes artères de mobilité répondait évidemment à la fonction dissuasive des lieux d'exécution, afin de décourager tout malintentionné venant d'ailleurs de se laisser tenter par le crime. Mais, comme nous venons de l'évoquer, le rôle de ces lieux allait au-delà de la dissuasion ou de l'exclusion sociale du malfaiteur, en participant par exemple à la construction des espaces politiques, ce qui leur confère un rôle essentiel dans la communication politique et de la mise en relation. 


\section{PRATIQUER L'ESPACE : LA SPATIALITÉ COMME RESSOURCE DU PÉNAL}

La peine investit elle-même l'espace - à nouveau, ce constat parait si évident qu'il faut s'étonner que bien peu d'études se soient intéressées de plus près à cette question. Ainsi, force est de constater que nous manquons cruellement d'une véritable histoire sociale des espaces de l'enfermement, lacune comblée seulement en partie par les travaux en histoire d'architecture pénale, souvent d'inspiration foucaldienne et focalisés majoritairement sur le XIX ${ }^{\mathrm{e}}$ siècle $^{18}$. Si les études spatiales que Foucault avait proposées dans Surveiller et punir restent stimulantes, elles se sont largement limitées à l'analyse d'un espace sériel - produit par les discours afin de permettre un maximum de contrôle et de surveillance par l'organisation d'une visibilité efficace et assujettissante - et non pas d'un espace vécu ou pratiqué ${ }^{19}$. De nombreux travaux ont été en revanche consacrés aux peines qui, pour déployer leurs effets d'exclusion, avaient recours à un espace à large échelle. C'est le cas des études portant sur l'histoire des galères, par exemple en France ou dans l'empire ottoman ${ }^{20}$, mais aussi de celles qui s'intéressent aux différentes formes d'expulsion ou de déportation, que ce soit vers l'outre-mer - sanction pratiquée par la quasi-totalité des puissances coloniales, du Portugal et de l'Espagne, jusqu'à l'Angleterre et la France, en passant par des exemples moins connus comme le Danemark ${ }^{21}$ - ou vers les contrées lointaines de la colonisation intérieure, comme dans le cas russe ${ }^{22}$. La grille de lecture que ces études appliquent à leur objet, cependant, ne confère que rarement à l'espace le rôle d'une catégorie d'analyse à part entière, si ce n'est pour inscrire la peine dans l'horizon plus large d'une entreprise de conquête spatiale, comme dans le cas de la fameuse transportation des condamnés anglais vers les colonies pénitentiaires en Australie ${ }^{23}$.

Le manque le plus étonnant concerne pourtant l'une des sanctions les plus fréquemment appliquées dans l'Europe moderne, à savoir le bannissement ${ }^{24}$. Cette absence frappe surtout dans le cas français où les rares études existantes ont en fait trait à des terrains germaniques ou slaves - et sont dues à des médiévistes ${ }^{25}$. Seule exception parmi les modernistes, Nicole Castan a consacré quelques remarques à cette sanction dans ses recherches portant sur le Languedoc. ${ }^{26}$ Le bannissement a ainsi été relégué dans l'arrière-cour d'une historiographie attirée davantage par les réformes pénales des Lumières (aux yeux desquelles cette peine n'a pas trouvé grâce)

18 Notamment Evans (1982); Dubbini (1986); Johnston (2000). Pour l'architecture carcérale de l'Italie médiévale Geltner (2008).

19 Foucault (1975, p. 143-151). Nous avons essayé nous-mêmes d'esquisser les premiers traits d'une autre histoire de ces espaces, voir Bretschneider (2014).

20 Zysberg (1987); Zarinebaf (2010, p. 164-168).

$21 \quad$ Pike (1983); Ekirch (1987); Coates (2002); Morgan et Rushton (2013); Anderson et Maxwell-Stewart (2013); Steiner (2014); de Vito et Lichtenstein (2015).

22 Kollmann (2012, p. 241-257).

23 Anderson (2016).

24 En revanche, une forme apparentée, le pèlerinage judiciaire, a été plus abondamment étudiée, notamment pour l'espace urbain des Pays-Bas: Rousseaux (1995, 2012).

25 Zaremska (1996) (traduit du polonais en français); Jacob (2000); rajoutons les quelques lignes, d'ordre plutôt général, consacrées à la peine par Gauvard (2007, p. 68-69).

26 Castan (1980, p. 266-298). Appuyé sur ces travaux, Castan (1984). 
ou fascinée par l'éclat des supplices ou d'autres châtiments corporels; il allait en outre à rebours du grand récit de la modernisation politique, celle qui passe par l'État, fort et centralisé, si bien qu'il s'est vu traiter de dernier vestige d'un «morcellement féodal $\aleph^{27}$ dont on veut faire croire qu'il était révolu depuis bien longtemps, alors qu'il continuait à déployer ses effets dans le domaine pénal ${ }^{28}$. La situation est légèrement différente aux Pays-Bas, en Italie ou pour l'espace germanique où nous disposons de quelques petites études sous forme d'articles ou de chapitres d'ouvrage ${ }^{29}$. Mais même sur ces terrains, on attend toujours un travail qui sache s'intéresser à ce qui peut être considéré comme l'essentiel du bannissement: le croisement entre, d'une part, les pratiques pénales au sein d'une forme d'organisation spatiale ne correspondant pas encore au modèle moderne de la territorialité «souveraine», et d'autre part, les effets récursifs qui résultaient de cette fragmentation de l'espace pour l'administration des peines et son appropriation par les condamnés ${ }^{30}$.

\section{S'APPROPRIER L’ESPACE: LES ESPACES DE LA JUSTICE}

De telles appropriations spatiales ne concernent pas seulement un certain type de condamnés mais aussi la justice elle-même, dans une plus large mesure. En suivant Joseph Morsel, on pourrait en distinguer plusieurs types ${ }^{31}:$ d'abord des appropriations «polaires» qui, en partant d'un point dans l'espace, marquent matériellement et symboliquement des espaces de la justice, à l'instar des lieux d'exécution évoqués plus haut; ensuite des appropriations «linéaires» qui tracent des frontières comme dans le cas du bannissement, en définissant un intérieur et un extérieur à la fois spatiaux et sociaux, mais aussi dans celui de la formation de la carte judiciaire, casse-tête séculaire ${ }^{32}$; puis des appropriations «aréolaires», destinées à manifester l'homogénéité d'un espace et mobilisées, par exemple, lors des grands spectacles punitifs et des processions qui les accompagnent ${ }^{33}$; enfin, des appropriations de «mise en réseau», par exemple par le jeu des références symboliques mobilisées lors des exécutions pénales et qui font leur, au-delà de chaque lieu concret, un espace de pratiques partagées.

Évidemment, ces appropriations de l'espace par la justice pénale provoquent à leur tour des pratiques plus ou moins subversives de contournement ou de transformation chez les acteurs. Ainsi, dès le Moyen Âge, les justiciables savent

27 Carbasse (2000, p. 289-292).

28 Voir par exemple la compilation de la jurisprudence relative à la peine que donne, à la fin du XVIII siècle, Guyot (1776).

29 Diederiks (1985); Schnabel-Schüle (1995); Marchal (1996); Gaulin (1997); Schwerhoff (2006); pour l'Italie, l'étude du bannissement recoupe en partie celle, beaucoup plus fournie, de l'exil politique. Voir Baxendale (1991) ou Shaw (2000).

30 C'est justement l'aspect délaissé par la seule étude monographique existante, celle de Coy (2008) qui porte sur Ulm, ville d'Empire; nous préparons actuellement un travail consacré à ces questions en partant des parties orientales du Saint-Empire.

31 Morsel (2006, p. 97-99)

32 Numéro «Territoires et lieux», Histoire de la justice (2011).

33 Evans (1996, p. 73-77); Bastien (2006, p. 122-129). 
tirer profit de l'imbroglio des juridictions ${ }^{34}$ ou, plus largement, d'une géographie des concurrences politiques et judiciaires qui leur confère parfois des marges de manœuvre importantes, comme quand ils peuvent choisir entre plusieurs types de justices, par exemple ecclésiastiques ou laïques ${ }^{35}$. Dans ce cas leurs choix se rapportaient à des critères économiques (taux des frais de procédure, etc.) mais davantage encore à des critères spatiaux: l'accessibilité des tribunaux et les possibilités de mobilité de ceux qui étaient censés les fréquenter, leur distance géographique et donc la longueur du déplacement (qui renvoie aux conditions économiques, car tout éloignement était synonyme de frais et de pertes de salaire, des aspects thématisés à nouveau, aujourd'hui, de manière différente, par les travaux autour des «justices spatiales»). Certes, ces diverses options valaient d'abord pour la justice civile, mais les criminels aussi savaient jouer du camaïeu des différents pouvoirs politiques et juridiques, notamment dans le Saint-Empire, par exemple en prenant la fuite tout en sachant que ceux qui étaient à leurs trousses seraient sérieusement freinés dans leur élan par l'inefficacité de la force publique et, davantage encore, par les effets spatiaux d'un droit qui rendait quasiment impossible d'effectuer une poursuite efficace supposant de transgresser les omniprésentes frontières des territoires ${ }^{36}$.

À ces quelques exemples, on pourrait en ajouter d'autres - par exemple l'histoire des espaces propres à la justice pénale, c'est-à-dire des lieux où elle s'exerce (bâtiments de tribunaux, partagés avec d'autres administrations ou dédiés à un usage unique $^{37}$ ), sujet proche de celui d'une histoire matérielle de la justice ${ }^{38}$. Là aussi, nos quelques remarques ne se veulent donc pas exhaustives mais souhaitent attirer l'attention sur un champ de recherche qui attend encore largement d'être défriché.

Falk Bretschneider Centre Georg Simmel Recherches franco-allemandes en sciences sociales - UMR 8131 bretschn@ehess.fr

\section{BIBLIOGRAPHIE}

Anderson, C. (2016) Transnational Histories of Penal Transportation: Punishment, Labour and Governance in the British Imperial World, 1788-1939, Australian Historical Studies, 47, 3, p.381-397.

Anderson, C. et Maxwell-Stewart, H. (2013) Convict Labour and the Western Empires, 14151954, in Aldrich, R et McKenzie, K. (Eds.), The Routledge History of Western Empires, Londres: Routledge, p. 102-117.

Association française d'histoire de la justice (1992) La Justice en ses temples: regards sur l'architecture judiciaire en France, Poitiers, Brissaud et Paris: Errance.

\footnotetext{
34 Gauvard (2007).

35 Ströhmer (2013).

36 Härter (2011).

37 Leuwers (2010, p. 57-62); Association française d'histoire de la justice (1992); Madranges (2011).

38 Porret et al. (2012).
} 
Auler, J. (2008-2012) Richtstättenarchäologie, 3 vol., Dormagen: Archeotopos.

Bachmann-Medick, D. (2006) Cultural Turns. Neuorientierungen in den Kulturwissenschaften, Reinbek: Rowohlt.

Bastien, P. (2006) L'exécution publique à Paris au XVIII' siècle. Une histoire des rituels judiciaires, Seyssel: Champ Vallon.

Baxendale, S.F. (1991) Exile in Practice: The Alberti Family In and Out of Florence 1401-1428, Renaissance Quarterly, 44, 4, p. 720-756.

Bellabarba, M. (2002) Zeugen der Macht: Adelige und tridentinische Bauerngemeinden vor den Richtern (16.-18. Jahrhundert), in Fuchs, R.-P. et Schulze, W. (Eds.), Wahrheit, Wissen, Erinnerung. Zeugenverhörprotokolle als Quellen für soziale Wissensbestände in der Frühen Neuzeit, Münster: Lit, p. 201-224.

Bourdelais, P. et Lepetit, B. (1986) Histoire et espace, in Auriac, F. et Brunet, R. (Dir.), Espaces, jeux et enjeux, Paris: Fayard, p. 17-26.

Bret, B., Gervais-Lambony, P., Hancock, C. et Landy, F. (Dir.) (2010) Justice et injustices spatiales, Nanterre: Presses universitaires de Paris Nanterre.

Bretschneider, F. (2014) Spaces of Confinement. Institutional Stabilization and Eigensinn - the Case of Saxony, in Friedrich, K., Bailey, G. et Veit, P. (Eds.), Die Erschließung des Raumes: Konstruktion, Imagination und Darstellung von Räumen und Grenzen im Barockzeitalter, Wolfenbüttel, Wiesbaden: Otto Harrassowitz, p.97-113.

Bretschneider, F. (2018) Étudier la fractalité: les espaces du Saint-Empire entre pluralité des échelles et liens transversaux, in Bretschneider, F. et Duhamelle, C. (Dir.), Le Saint-Empire, histoire sociale (XVI ${ }^{e}$-XVIII ${ }^{e}$ siècles), Paris: Éditions de la Maison des sciences de l'homme, p. 147-165.

Bretschneider, F. et Duhamelle, C. (2016) Fraktalität. Raumgeschichte und soziales Handeln im Alten Reich, Zeitschrift für historische Forschung, 43, 4, p. 703-746.

Brizay, F., Follain, A. et Sarrazin, V. (Dir.) (2002) Les justices de village. Administration et justice locales de la fin du Moyen Âge à la Révolution, Rennes: Presses universitaires de Rennes.

Bubenicek, M. (2015) «Et encourt sont les dictes forches en la dicte place toutes droictes...». La guerre des gibets dans l'État bourguignon naissant (Franche-Comté, 1380-1400), in actes de colloques et de journées d'études Criminicorpus, Les Fourches Patibulaires du Moyen Âge à l'Époque moderne. Approche interdisciplinaire, [En ligne] http://criminocorpus. revues.org/3040.

Carbasse, J.-M. (2000), Histoire du droit pénal et de la justice criminelle, Paris: PUF.

Castan, N. (1980) Justice et répression en Languedoc à l'époque des Lumières, Paris: Flammarion.

Castan, Y. (1984) Exil ou prison en Languedoc au XVIII e siècle, in Petit, J. G. (Dir.), La prison, le bagne et l'histoire, Genève: Médecine et hygiène, p. 57-66.

Charageat, M. et Vivas, M. (2015) Les fourches patibulaires du Moyen Âge à l'époque moderne. Approche interdisciplinaire, in actes de colloques et de journées d'études Criminicorpus, [En ligne] http://criminocorpus.revues.org/3018.

Chauvaud, F., Jean, Y. et Willemez, L. (Dir.) (2001) Justice et sociétés rurales, du XVI siècle à nos jours: approches pluridisciplinaires, Rennes: Presses universitaires de Rennes.

Coates, T. (2002) Convicts and orpahns. Forced and State-Sponsored Colonizers in the Portuguese Empire, 1550-1755, Stanford: Stanford University Press. 
Coy, J.P. (2008) Strangers and Misfits. Banishment, Social Control, and Authority in Early Modern Germany, Leiden \& Boston: Brill.

De Vito, C.G. et Lichtenstein, A. (Dir.) (2015) Global Convict Labor, Leiden \& Boston: Brill.

Denys, C. (2016) Geography of Crime. Urban and Rural Environments, in Knepper, P. et Johansen, A. (Eds.), The Oxford Handbook of the History of Crime and Criminal Justice, Oxford: Oxford University Press, p. 88-108.

Diederiks, H. (1985) Stadt und Umland im Lichte der Herkunftsorte der Kriminellen in Leiden im 17. und 18. Jahrhundert, in Schulze, H.K. (Dir.), Städtisches Um- und Hinterland in vorindustrieller Zeit, Köln: Böhlau, p. 183-205.

Döring, J. et Thielmann, T. (2008) Spatial Turn. Das Raumparadigma in den Kultur- und Sozialwissenschaften, Bielefeld: Transcript.

Dubbini, R. (1986) Architettura delle prigioni: i luoghi e il tempo della punizione, 1700-1880, Milan: Franco Angeli.

Dyndor, Z. (2015) The Gibbet in the Landscape: Locating the Criminal Corpse in MidEighteenth-Century England, in Ward, R. (Ed.), A Global History of Execution and the Criminal Corpse, Basingstoke: Palgrave Macmillan, p. 102-125.

Ekirch, A.R. (1987) Bound for America. The transportation of British convicts to the colonies, 1718-1775, Oxford: Clarendon Press.

Evans, R. (1982) The Fabrication of Virtue: English Prison Architecture, 1750-1840, Cambridge: Cambridge University Press.

Evans, R.J. (1996) Rituals of Retribution: Capital Punishment in Germany, 1600-1987, Oxoford: Oxford University Press.

Foucault, M. (1975) Surveiller et punir. Naissance de la prison, Paris: Gallimard.

Frank, M. (1995) Dörfliche Gesellschaft und Kriminalität: das Fallbeispiel Lippe 1650-1800, Paderborn: Schöningh.

Gaulin, J.L. (1997) Les registres de bannis pour dettes à Bologne au XIII' siècle: une nouvelle source pour l'histoire de l'endettement, Mélanges de l'École française de Rome. Moyen Âge, 109, 2, p. 479-499.

Gauvard, C. (2007) Droit et pratiques judiciaires dans les villes du nord du royaume de France à la fin du Moyen Âge. L'enseignement des sources, in Chiffoleau, J., Gauvard, C. et Zorzi, A. (Eds.), Pratiques sociales et politiques judiciaires dans les villes de l'Occident à la fin du Moyen Âge, Rome: École française de Rome, p. 33-79.

Geltner, G. (2008) The Medieval Prison: A Social History, Princeton: Princeton University Press.

Guyot, J.-N. (dir.) (1776) Répertoire universel et raisonné de jurisprudence civile, criminelle, canonique et bénéficiale, Paris: Panckoucke.

Härter, K. (2011) Die Formierung transnationaler Strafrechtsregime. Auslieferung, Asyl und grenzübergreifende Kriminalität im Übergang von gemeinem Recht zum nationalstaatlichen Strafrecht, Rechtsgeschichte, 18, p. 36-65.

Jacob, C. (2014) Spatial turn, in Jacob, C. (Ed.) Qu'est-ce qu'un lieu de savoir ?, [En ligne] http://books.openedition.org/oep/654.

Jacob, R. (2000) Bannissement et rite de la langue tirée au Moyen Âge: du lien des lois et de sa rupture, Annales. Histoire, Sciences Sociales, 55, 5, p. 1039-1079. 
Johnston, N.B. (2000) Forms of Constraint: A History of Prison Architecture, Chicago: University of Illinois Press.

Justice spatiale (2009) Annales de géographie, 665-666, 1, p. 1-168.

Kollmann, N.S. (2012) Crime and punishment in early modern Russia, Cambridge: Cambridge University Press.

Lepetit, B. (1999) Espace et histoire, in Lepetit, B. (Ed.), Carnet de croquis. Sur la connaissance historique, Paris: Albin Michel, p. 129-141.

Leuwers, H. (2010) La justice dans la France moderne. Du roi de justice à la justice de la nation (1498-1792), Paris: Ellipses Edition.

Löw, M. (2015) Sociologie de l'espace, Paris: Éditions de la Maison des sciences de l'homme (éd. allemande 2001).

Madranges, E. (2011) Les palais de justice de France, Paris: LexisNexis.

Marchal, G.P. (1996) Von der Stadt und bis ins Pfefferland. Städtische Raum- und Grenzvorstellungen in Urfehden und Verbannungsurteilen oberrheinischer und schweizerischer Städte, in Marchal, G.P. (Ed.), Grenzen und Raumvorstellungen (11.-20. Jh.), Zurich: Chronos, p. 225-263.

Morgan, G. et Rushton, P. (2013) Banishment in the Early Atlantic World. Convicts, Rebels and Slaves, Londres: Bloomsbury.

Morsel, J. (2006) Appropriation communautaire du territoire, ou appropriation territoriale de la communauté? Observations en guise de conclusion, Hypothèses, 9, 1, p. 89-104.

Pike, R. (1983) Penal Servitude in Early Modern Spain, Madison: University of Wisconsin Press.

Porret, M., Montana, V. et Magué, L. (2012) Bois, fers et papiers de justice. Histoire matérielle du droit de punir, Genève: Georg.

Rau, S. (2008) Räume, Francfort/M.: Campus.

Revel, J. (1999) Jeux d'échelles. La micro-analyse à l'expérience, Paris: Gallimard-Le Seuil.

Rousseaux, X. (1995) Partir ou payer? Le pèlerinage judiciaire à Nivelles (XV'-XVII ${ }^{\mathrm{e}}$ siècles), in Dauchy, S. et Sueur, P. (Eds.), La route. Actes des Journées d'histoire du droit d'Enghien, Lille: CHJ Éditeur, p. 105-140.

Rousseaux, X. (2012) Religion, économie et société. Le pèlerinage judiciaire dans les Pays-Bas (Nivelles, du XV ${ }^{\mathrm{e}}$ au XVII ${ }^{\mathrm{e}}$ siècle), in Bourguignon, M.-A., Dauven, B. et Rousseaux, X. (Eds.), Amender, sanctionner et punir. Recherches sur l'histoire de la peine du Moyen Âge au $X X^{e}$ siècle, Louvain:Presses universitaires de Louvain, p. 61-85.

Schnabel-Schüle, H. (1995) Die Strafe des Landesverweises in der Frühen Neuzeit, in Gestrich, A., Hirschfeld, G. et Sonnabend, H. (Eds.), Ausweisung und Deportation. Formen der Zwangsmigration in der Geschichte, Stuttgart: Steiner, p. 73-82.

Schuster, P. (2015) Verbrecher, Opfer, Heilige. Eine Geschichte des Tötens 1200-1700, Stuttgart: Klett-Cotta.

Schwerhoff, G. (2006) Vertreibung als Strafe. Der Stadt- und Landesverweis im Ancien Régime, in Hahn, S., Komlosy, A. et Reiter, I. (Eds.), Ausweisung, Abschiebung, Vertreibung in Europa. 16.-20. Jahrhundert, Innsbruck: Studienverlag, p. 48-72.

Shaw, C. (2000) The Politics of Exile in Renaissance Italy, Cambridge: Cambridge University Press.

Soja, E. (1996) Thirdspace: Journeys to Los Angeles and Other Real-and-imagined Places, Malden: Blackwell. 
Spierenburg, P. (1984) The Spectacle of Suffering. Executions and the evolution of repression: from a preindustrial metropolis to the European experience, Cambridge: Cambridge University Press.

Steiner, S. (2014) Rückkehr unerwünscht. Deportationen in der Habsburgermonarchie der Frühen Neuzeit und ihr europäischer Kontext, Vienne: Böhlau.

Ströhmer, M. (2013) Jurisdiktionsökonomie im Fürstbistum Paderborn. Institutionen Ressourcen - Transaktionen (1650-1800), Münster: Aschendorff.

Territoires et lieux de justice (2011) Histoire de la justice, 21.

Warf, B. et Arias, S. (Dir.) (2009) The spatial turn: interdisciplinary perspectives, Londres: Routledge.

Zaremska, H. (1996) Les bannis au Moyen Âge, Paris: Aubier.

Zarinebaf, F. (2011) Crime and Punishment in Istanbul, 1700-1800, Berkeley: University of California Press.

Zotz, T. (2006) Présentation et bilan de l'historiographie allemande de l'espace, actes du $37^{e}$ congrès de la Société des historiens médiévistes de l'enseignement supérieur public (Mulhouse), Construction de l'espace au Moyen Âge: pratiques et representations, Paris: Publications de la Sorbonne, p.57-71.

Zysberg, A. (1987) Les galériens. Vies et destins de 60000 forçats sur les galères de France 1680-1748, Paris: Le Seuil. 